\title{
A Mathematical Model for ECG Waveform using Rational Bézier Curves and Bernstein Polynomials
}

\author{
V.Chutchavong, K.Nualon, O. Sangaroon \\ Dept. of Computer Engineering, Faculty of Engineering, \\ King Mongkut's Institute of Technology Ladkrabang \\ Bangkok, THAILAND \\ kcvanvis@kmitl.ac.th
}

\begin{abstract}
This paper presents a mathematical model for ECG waveform using Rational Bezier curves and Bernstein polynomials. There are three mathematical models; the Bernstein polynomials model, the Bézier-Bernstein model and the Rational Bézier-Bernstein model. All mathematical models can generate the ECG waveform which is similar to the normal ECG waveform. Moreover, all mathematical models can simulate a normal ECG waveform as well. As the results, the Rational Bezier-Bernstein model is a simple form, easy to make a circuit and lower cost in the implementation of the ECG waveform.
\end{abstract}

Keywords-Bernstein polynomials; Bézier curves; ECG; Rational Bézier curves

\section{INTRODUCTION}

The electrocardiogram (ECG) is a electrical activity of the heart describes the cardiac cycle which is commonly used in medical and health care monitoring. The ECG is revealed via ECG machines which requires high accuracy and frequent calibration. Besides, the ECG simulation must be used for monitoring this machine. The ECG simulation requires more precision, similar to a normal ECG waveform and lowest complexity to implement ECG waveform. Many literatures have proposed the methods to generate the ECG simulation. The mathematical modeling of electrocardiograms by using numerical methods has been proposed in [1]. Reference [2] has presented a new ECG identification with Neural Network. S. Mitra [3], Hilbert transform, variable threshold and slope reversal has been implemented to the ECG feature. A Novel Bézier curve [4] and Karnel function [5] have been proposed for designing the mathematical model of an ECG waveform. Therefore, the ECG simulation must be generated with high precision before using the ECG machine.

This paper presents the mathematical model of electrocardiograms. There are three mathematical models; the Bernstein polynomials model, the Bézier-Bernstein model and the Rational Bézier-Bernstein model. All mathematical models can generate the ECG waveform which is similar to the normal ECG waveform. The rest of the paper is organized as follows. In section 2, we described the ECG waveform. The whole mathematical models are presented in section 3 . Then, section 4 shows the results and discussion. Finally, the conclusion is given in section 5 .

\author{
K. Janchitrapongvej \\ Faculty of Science and Technology, \\ Southeast Bangkok College \\ Bangkok, THAILAND \\ kjkanok@gmail.com
}

\section{ECG WAVEFORM}

The fact that nowadays failure of the heart has been analyzed by the electrocardiogram (ECG) waveform, especially cardiovascular disease diagnosis. Therefore, the ECG detection can diagnose the conditions of the patients. An ECG is a electrical activity of the heart describes the cardiac cycle. ECG machine using for the ECG waveform measurements required high accuracy and regular calibration by using an ECG simulator before using this machine [6]. An ECG waveform collects from sample people, saved in memory, called the normal ECG as shown in Table 1. [7][8]

TABLE I. EX. THE NORMAL ECG DATA

\begin{tabular}{|l|l|}
\hline Feature & \multicolumn{1}{|c|}{ Data of Normal ECG Waveform } \\
\hline \multirow{3}{*}{ P Wave } & 484848484848484848484848494949505254 \\
& 565860626465656666656563626159585655 \\
& 545352525151505049494949494948484949 \\
\hline \multirow{3}{*}{ QRS } & 484846423834312927283137486588108135 \\
Complex & 14615215414513712811710796847054342522 \\
& 222426303336384042434445464647474747 \\
\hline \multirow{4}{*}{ T Wave } & 47484848 \\
& 596848484848484949494949505152535557 \\
& 787572696563605855535251515050504949 \\
\hline
\end{tabular}

Table 1 shows the example of the data of the normal ECG waveform which is shown in Fig.1.

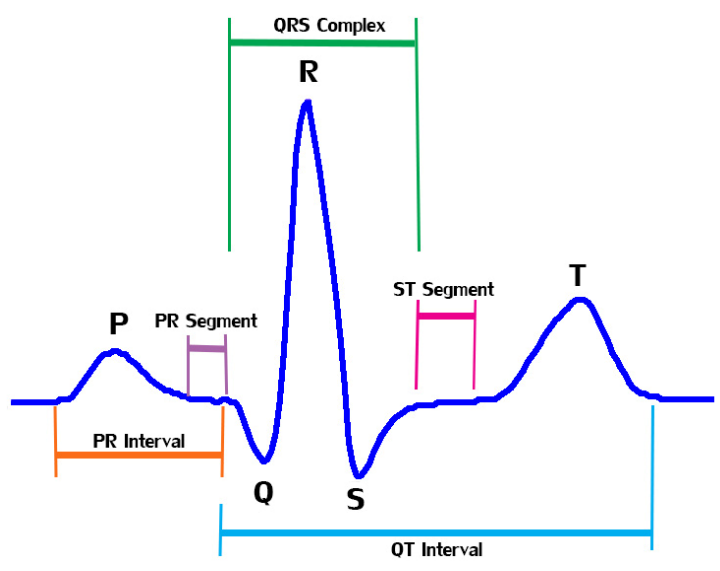

Fig. 1. The normal ECG Waveform. 
Fig.1 shows the normal ECG waveform of the cardiac cycle which was simulated from the normal ECG data in Table 1. It can be seen that the normal ECG waveform consists of a P wave, a QRS complex and a T wave (U wave doesn't consider since it has lower amplitude and is hidden by $\mathrm{T}$ wave). In a normal healthy heart, the representative of the periods in the cardiac cycle. Furthermore, this paper has proposed the development of mathematical models for generating the ECG simulation. The mathematical modeling is described in the next section.

\section{MATHEMATICAL MODELS}

\section{A. Bernstein Polynomials Model}

The Bernstein polynomials of degree $n^{\text {th }}$ are defined by

$$
B_{i}^{n}(t)=\left(\begin{array}{c}
n \\
i
\end{array}\right) t^{i}(1-t)^{n-i}
$$

The Bernstein polynomials arise in approximation theory when we desire to find a uniform approximation by polynomials to continuous functions over a closed interval. This is given by [9][10]

$$
B_{i}^{n}(f ; t)=\sum_{i=0}^{n} f\left(\frac{i}{n}\right)\left(\begin{array}{c}
n \\
i
\end{array}\right) t^{i}(1-t)^{n-i}
$$

where $i=0,1, \ldots, n$ and $\left(\begin{array}{l}n \\ i\end{array}\right)=\frac{n !}{i !(n-i) !}$

Equation (2) has been used to approximate a continuous function $f(t)$ in the interval [0,1]. A continuous function is considered the approximation of the normal ECG waveform as shown in Fig. 2.

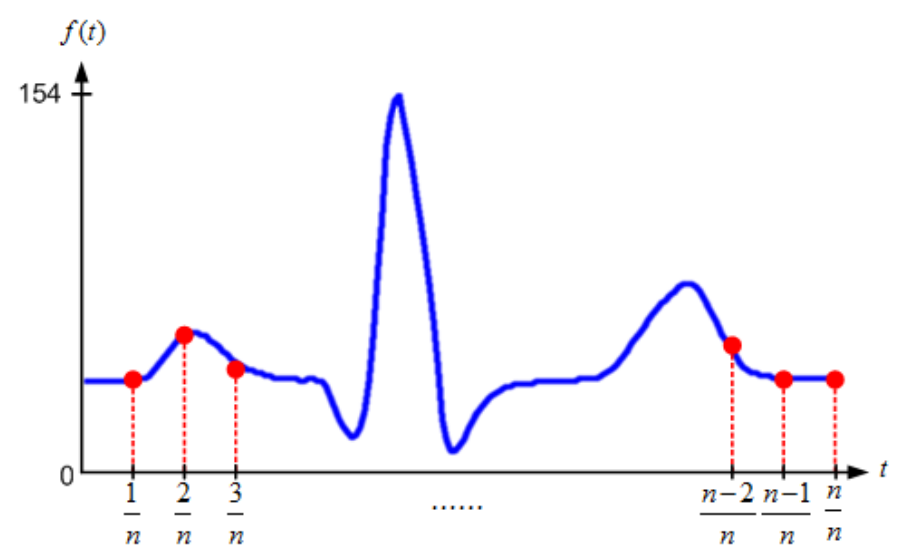

Fig. 2. Approximation of a continuous function.

Fig. 2 shows the approximation of a continuous function as the normal ECG waveform. The number of successive discrete points $(i / n)$ is equal to $n$ that begins with $i=0(0 / n)$ to $i=1(n / n)$, so it is always in the interval $[0,1]$. In order to find the Bernstein polynomials of degree $n^{\text {th }}$ for approximating the normal ECG waveform. In the simulation, let us assume the parameter $n=25, n=100, n=200$ and $n=1000$ and then replacing into Eq.(1) as shown in Fig. 3(a), Fig. 3(b), Fig. 3(c) and Fig. 3(d), respectively.

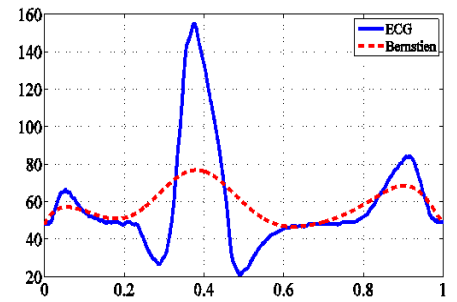

(a) $n=25$.

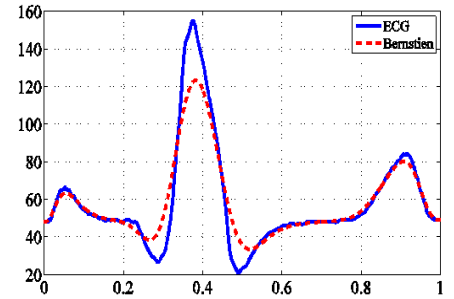

(c) $n=200$

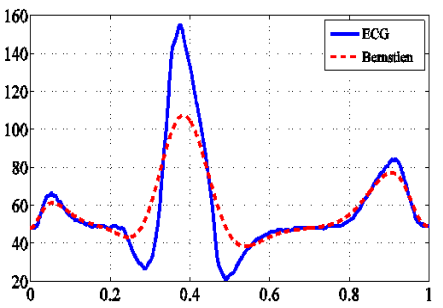

(b) $n=100$.

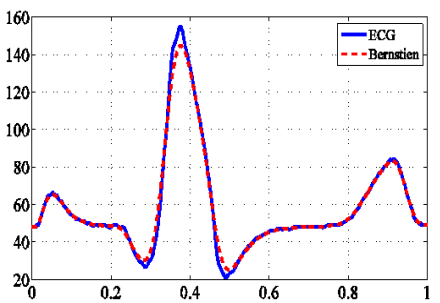

(d) $n=1000$.
Fig. 3. A comparison between the normal ECG waveform and the ECG simulation by using Bernstein polynomials model with varying $n$.

From Fig. 3(a) shows the comparison between the normal ECG waveform and the ECG simulation with $n=25$. It can be seen that the ECG simulation (red line) is different from the normal ECG waveform (blue line) or it has many errors. Thus, the simulation must increase the value of $n$. Fig. 3(b) and Fig. 3(c) illustrate the comparison between the normal ECG waveform and the ECG simulation with $n=100$ and $n=200$, respectively. It stated that the ECG simulation is nearly the normal ECG waveform or the errors is less than Fig. 3(a). Fig. 3(d) shows the comparison between the normal ECG waveform and the ECG simulation with $n=1000$. It can be concluded that the both waveforms is similar. Therefore, using the Bernstein polynomials model at degree $n^{\text {th }}$ requires an extremely high order which makes the difficult form, the more complicated design, and higher costs.

\section{B. Bézier-Bernstein Model}

There is the way to formulate Bézier curves mathematically that is the Bernstein polynomials, called the Bézier-Bernstein curves. The Bernstein polynomials form of Bézier curves is given by [11][12][13]

$$
B(t)=\sum_{i=0}^{n} p_{i} B_{i}^{n}(t)
$$

where $B_{i}^{n}(t)$ is the Bernstein polynomials as Eq.(1). $p_{i}$ are the points of control curves, $p_{i}=\left\{p_{0}, p_{1}, p_{2}, \ldots, p_{n}\right\}$.

The Bézier curves usually define the curve for $[0,1]$. This interval is as same as the Bernstein polynomials. Considering the approximation of the normal ECG waveform which can be 
divided into three intervals. Firstly, the $\mathrm{P}$ wave is approximated by Bézier-Bernstein model that starts by letting the five points of a control $\mathrm{P}$ wave. Given five control points are $P_{x}=\left\{p_{0}=1, p_{1}=14, p_{2}=26, p_{3}=42, p_{4}=54\right\} \quad$ for the horizontal axis (x-axis) and $P_{y}=\left\{p_{0}=48, p_{1}=49, p_{2}=66\right.$, $\left.p_{3}=51, p_{4}=49\right\}$ for the vertical axis (y-axis). Secondly, the QRS complex is also given five control points. These control points are $P_{x}=\left\{p_{0}=57, p_{1}=65, p_{2}=74, \quad p_{3}=86, p_{4}=97\right\}$ for the horizontal axis and $P_{y}=\left\{p_{0}=46, p_{1}=31, p_{2}=154\right.$, $\left.p_{3}=22, p_{4}=44\right\}$ for the vertical axis. Finally, the $\mathrm{T}$ wave is also given five control points. These control points are $P_{x}=\left\{p_{0}=109, p_{1}=122, p_{2}=141, p_{3}=156, p_{4}=174\right\}$ for the horizontal axis and $P_{y}=\left\{p_{0}=48, p_{1}=51, p_{2}=84, p_{3}=51\right.$ $\left.p_{4}=49\right\}$ for the vertical axis. The position of the whole points determines the $\mathrm{P}$ wave, the QRS complex and $\mathrm{T}$ wave are shown in Fig. 4.

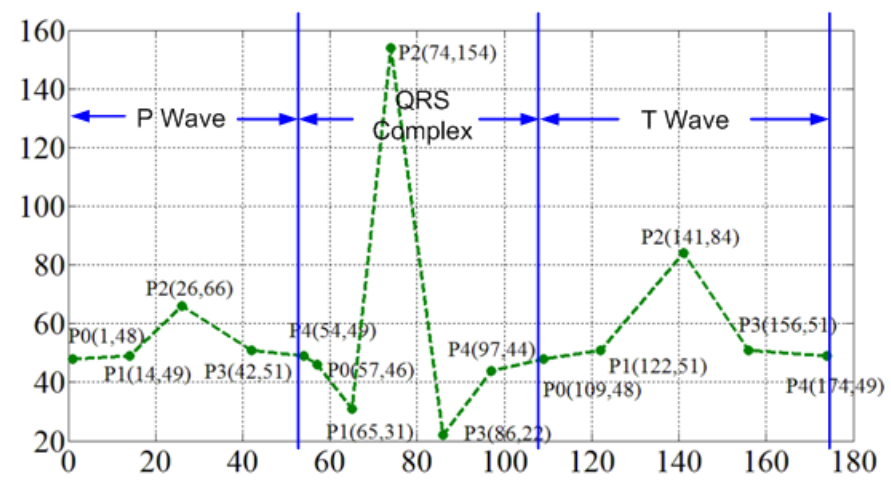

Fig. 4. The position of control points.

Fig. 4 shows the position of the whole points determines the normal ECG wave. From Eq. (3) can be expanded by using the five control points, given by

$$
\begin{gathered}
B(t)=B_{0}^{4}(t) p_{0}+B_{1}^{4}(t) p_{1}+B_{2}^{4}(t) p_{2}+B_{3}^{4}(t) p_{3}+B_{4}^{4}(t) p_{4} \\
B(t)=p_{0}(1-t)^{4}+p_{1} 4 t(1-t)^{3}+p_{2} 6 t^{2}(1-t)^{2}+p_{3} 4 t(1-t)+p_{4} t^{4}
\end{gathered}
$$

Consequently, the Bézier-Bernstein model of the normal ECG waveform is obtained as follows.

For P wave,

$$
\begin{gathered}
B_{x}(t)=(1-t)^{4}+56 t(1-t)^{3}+156 t^{2}(1-t)^{2}+168 t^{3}(1-t)+54 t^{4} \\
B_{y}(t)=48(1-t)^{4}+196 t(1-t)^{3}+396 t^{2}(1-t)^{2}+204 t^{3}(1-t)+49 t^{4}
\end{gathered}
$$

For QRS Complex,

$$
B_{x}(t)=57(1-t)^{4}+260 t(1-t)^{3}+444 t^{2}(1-t)^{2}+344 t^{3}(1-t)+97 t^{4}
$$

$$
B_{y}(t)=46(1-t)^{4}+1244 t(1-t)^{3}+924 t^{2}(1-t)^{2}+88 t^{3}(1-t)+44 t^{4}
$$

For T wave,

$$
B_{x}(t)=109(1-t)^{4}+488 t(1-t)^{3}+864 t^{2}(1-t)^{2}+624 t^{3}(1-t)+174 t^{4}
$$

$$
B_{y}(t)=48(1-t)^{4}+204 t(1-t)^{3}+504 t^{2}(1-t)^{2}+204 t^{3}(1-t)+49 t^{4}
$$

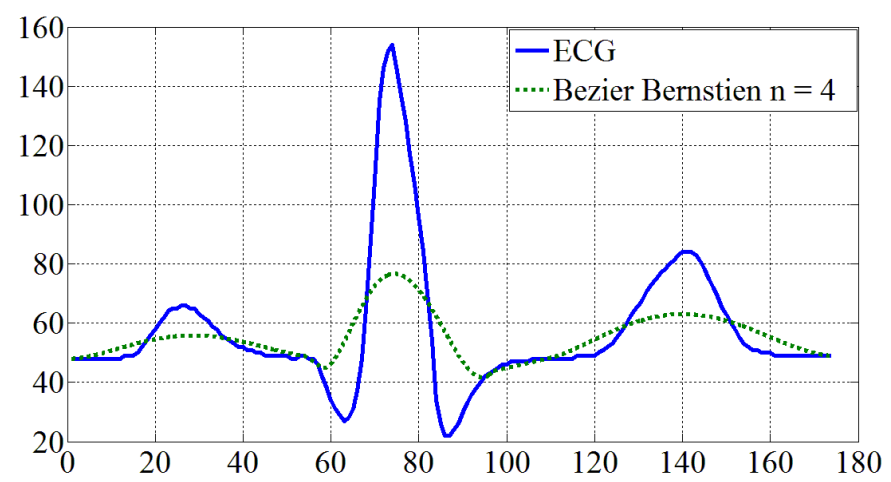

Fig. 5. A comparison between the normal ECG waveform and the ECG simulation by using Bézier-Bernstein curves model with $n=4$.

Fig. 5 shows a comparison between the normal ECG waveform and the ECG simulation by using Bézier-Bernstein curves model with $n=4$. Note that the ECG simulation (green line) is not identical to the normal ECG waveform (blue line); therefore, the Bézier-Bernstein model must increase the control points as shown in Fig. 6.

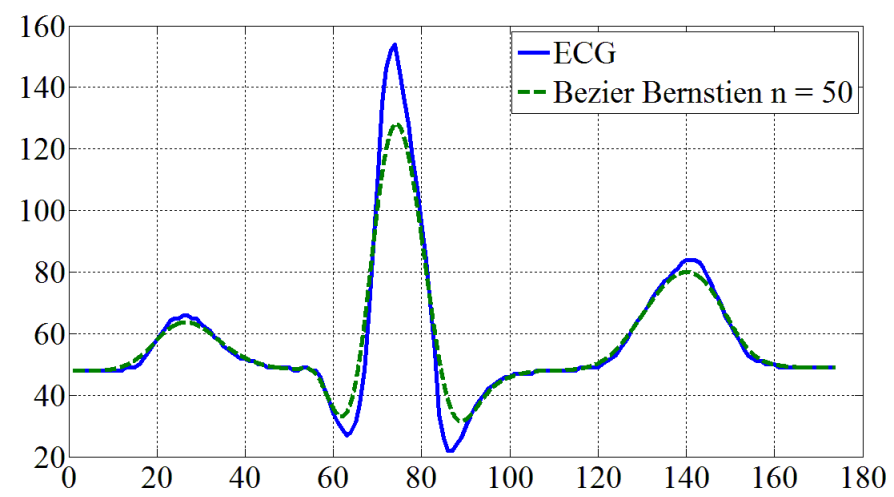

Fig. 6. A comparison between the normal ECG waveform and the ECG simulation by using Bézier-Bernstein model with $n=50$.

Fig. 6 shown a comparison between a normal ECG waveform and the ECG simulation by using Bézier-Bernstein curves model with $n=50$. It is seen that the ECG simulation is close to the normal ECG waveform. However, using the Bézier-Bernstein model have to exclusively determine the optimum value and the number of control points. The large number of control points becomes a problem of the Bézier- 
Bernstein model because it will affect the complexity of implementation.

\section{Ration Bézier-Bernstein Polynomials Model}

In order to effectively design a mathematical model and reduce the computational complexity of the control points, by using another method, the Rational Bézier-Bernstein curve are proposed. There are several advantages. For example, it can represent a wide range of curves and surfaces. Moreover, it has capable of representing the various forms such as circles, ellipses, parabolas and hyperbolas. The Rational BézierBernstein curves of degree $n^{\text {th }}$ becomes [11] [12] [13] [14]

$$
R(t)=\frac{\sum_{i=0}^{n} p_{i} w_{i} B_{i}^{n}(t)}{\sum_{i=0}^{n} w_{i} B_{i}^{n}(t)}
$$

where $w_{i}$ are the weights corresponding to the control points $\left(p_{i}\right)$ for $i=0,1, \ldots, n$. From Eq. (12) can be expanded by using the five control points, given by

$$
R(t)=\frac{B_{0}^{4}(t) p_{0} w_{0}+B_{1}^{4}(t) p_{1} w_{1}+B_{2}^{4}(t) p_{2} w_{2}+B_{3}^{4}(t) p_{3} w_{3}+B_{4}^{4}(t) p_{4} w_{4}}{B_{0}^{4}(t) w_{0}+B_{1}^{4}(t) w_{1}+B_{2}^{4}(t) w_{2}+B_{3}^{4}(t) w_{3}+B_{4}^{4}(t) w_{4}}
$$

The first step, we determine the control points the same as the previous section. There are five control points for each of the curve; the $\mathrm{P}$ wave, the QRS complex and the T wave. In the second step, let us assume the value of the weights. Given the weights are $w_{0}=0.25, w_{1}=100, w_{2}=1000, w_{3}=100, w_{4}=0.25$ for $\mathrm{P}$ wave, $w_{0}=0.5, w_{1}=200, w_{2}=1500, w_{3}=100, w_{4}=0.25$ for QRS complex and $w_{0}=0.15, w_{1}=100, w_{2}=1000, w_{3}=200$, $w_{4}=0.15$ for $\mathrm{T}$ wave. Therefore, the Rational BézierBernstein model of the normal ECG waveform is obtained as follows.

For P wave

$R_{x}(t)=\frac{0.25(1-t)^{4}+5600 t(1-t)^{3}+156000 t^{2}(1-t)^{2}+16800 t^{3}(1-t)+13.5 t^{4}}{0.25(1-t)^{4}+100 t(1-t)^{3}+1000 t^{2}(1-t)^{2}+100 t^{3}(1-t)+0.25 t^{4}}$

$R_{y}(t)=\frac{12(1-t)^{4}+19600 t(1-t)^{3}+396000 t^{2}(1-t)^{2}+20400 t^{3}(1-t)+12.25 t^{4}}{0.25(1-t)^{4}+100 t(1-t)^{3}+1000 t^{2}(1-t)^{2}+100 t^{3}(1-t)+0.25 t^{4}}$

For QRS complex

$R_{x}(t)=\frac{28.5(1-t)^{4}+52000 t(1-t)^{3}+666000 t^{2}(1-t)^{2}+34400 t^{3}(1-t)+24.25 t^{4}}{0.5(1-t)^{4}+200 t(1-t)^{3}+1500 t^{2}(1-t)^{2}+100 t^{3}(1-t)+0.25 t^{4}}$
$R_{y}(t)=\frac{23(1-t)^{4}+24800 t(1-t)^{3}+1386000 t^{2}(1-t)^{2}+8800 t^{3}(1-t)+11 t^{4}}{0.5(1-t)^{4}+200 t(1-t)^{3}+1500 t^{2}(1-t)^{2}+100 t^{3}(1-t)+0.25 t^{4}}$

For T wave

$R_{x}(t)=\frac{16.35(1-t)^{4}+48800 t(1-t)^{3}+846000 t^{2}(1-t)^{2}+124800 t^{3}(1-t)+26.1 t^{4}}{0.15(1-t)^{4}+100 t(1-t)^{3}+1000 t^{2}(1-t)^{2}+200 t^{3}(1-t)+0.15 t^{4}}$

$R_{y}(t)=\frac{7.2(1-t)^{4}+20400 t(1-t)^{3}+504000 t^{2}(1-t)^{2}+40800 t^{3}(1-t)+7.35 t^{4}}{0.15(1-t)^{4}+100 t(1-t)^{3}+1000 t^{2}(1-t)^{2}+200 t^{3}(1-t)+0.15 t^{4}}$

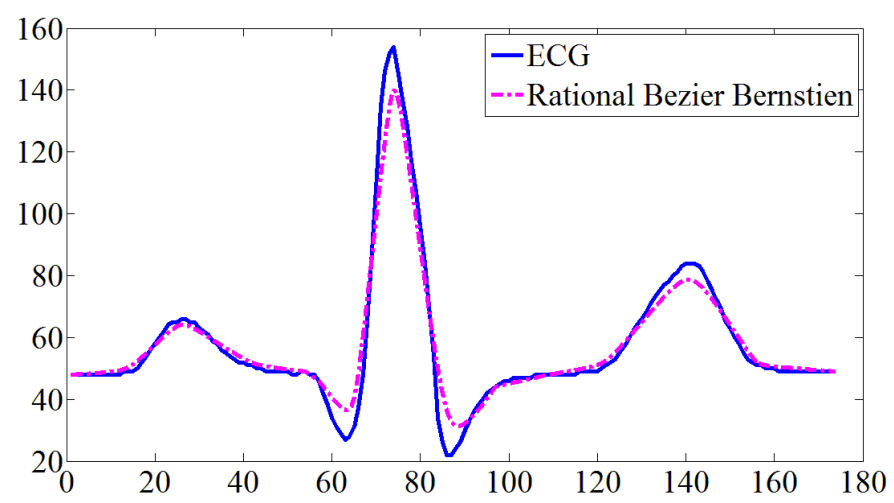

Fig. 7. A comparison between the normal ECG waveform and the ECG simulation by using Ration Bézier-Bernstein curves with $n=4$.

Fig. 7 shown a comparison between the normal ECG waveform and the ECG simulation by using Rational BézierBernstein model with $n=4$. It indicates that the ECG simulation is similar to the normal ECG waveform. Furthermore, the Rational Bézier-Bernstein model is quite simple form and easy to make the circuit or easy to implement the ECG waveform.

\section{RESULTS AND DisCUSSION}

This paper presents the mathematical models for the ECG waveform by using the Bernstein polynomials model, the Bézier-Bernstein model and the Rational Bézier-Bernstein model. As the simulation results, the proposed mathematical models can simulate the ECG waveform that is similarly the normal ECG waveform. This section discussed the simulation results of all three models. The first model which is the Bernstein polynomials can simulate the ECG waveform perfectly. Nevertheless, the degree of $n^{\text {th }}$ or order must be selected more than 200. From Fig. 3, the ECG simulation waveform with $n=1000$ is very perfectly but it has extremely order and difficult form. These disadvantages affect to make a circuit more complicated and higher cost in the implementation of the ECG waveform. The second model which is the BézierBernstein curves can reduce order remaining until 50. This model must determine the appropriated control points in both the horizontal axis and vertical axis. There are fifty-one control 
points for each of the curves; $\mathrm{P}$ wave, QRS complex and T wave. If the order is less than 50, then the ECG simulation is not same as the normal ECG waveform such as order 5 (shown in Fig. 5). From Fig. 6, the ECG simulation waveform with $n=50$ and the normal ECG waveform is similar. Thus, the percentage decrease order from $100 \% \quad(n=200)$ in the Bernstein polynomials model to $25 \%$ in the Bézier-Bernstein curves model. Moreover, it has still higher order, so the Rational Bézier-Bernstein model is proposed. The last model is the Rational Bézier-Bernstein curves which are lower order than other models. This model has an additional step which is the determination of the weights corresponding to the control points. For the simulation ECG waveform, the five weights corresponding to the five control points are determined for each of the curves; P wave, QRS complex and T wave. From Fig. 7, the ECG simulation waveform with $n=4$ just like the normal ECG waveform. Therefore, the percentage decrease order from $25 \%$ in the Bézier-Bernstein curves model to $2.5 \%$ in the Rational Bézier-Bernstein model. It can be seen that it is a simple form, easy to make a circuit and lower cost in the implementation of the ECG waveform.

A comparison between the normal ECG waveform and the three ECG simulation waveforms; the Bernstein polynomials model with $n=200$, the Bézier-Bernstein model with $n=50$ and the Rational Bézier-Bernstein model with $n=4$ are shown in Fig. 8.

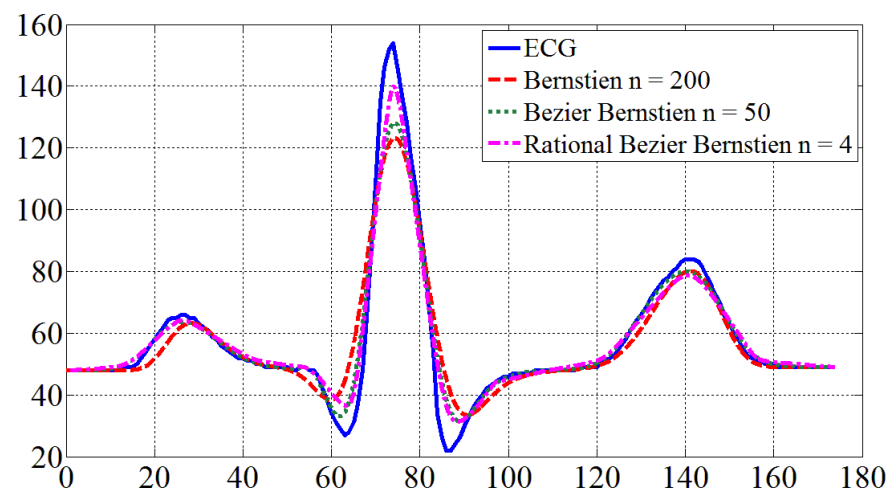

Fig. 8. A comparison of the ECG simulation between the Bernstein polynomials model, the Bézier-Bernstein curves model and the Ration BézierBernstein curves model.

From Fig. 8, it demonstrates that the whole ECG simulation waveform is the same as the normal ECG waveform; however, the degree of $n^{\text {th }}$ is extremely different order. To sum up, the Rational Bézier-Bernstein curve is the best mathematical modeling.

\section{CONCLUSION}

The mathematical models have been presented in order to generate the ECG simulation waveform. There are three mathematical models; the Bernstein polynomials model, the Bézier-Bernstein model and the Rational Bézier-Bernstein model. From the simulation results, all three models can generate the ECG simulation waveform similar to the normal
ECG waveform. In spite of the complication of mathematical models became an important problem. The Bernstein polynomials model used the extreme highest order which is to exceed 200. Next, the Bézier-Bernstein model used to be exceed 50. Then, the Rational Bézier-Bernstein curve model used lower order than other models. The Rational BézierBernstein model with $n=4$ can generate the ECG simulation waveform just like the normal ECG waveform. As the results, the Rational Bézier-Bernstein model is the simplest form to compare the Bernstein polynomials model and the BézierBernstein model. In addition, the simple form is easy to make a circuit and lower cost in the implementation of the ECG waveform.

\section{REFERENCES}

[1] M. Boulakia, S. Cazeau, M. A. Fernandez, J. F. Gerbeau, and N. Zemzemi, "Mathematical Modeling of Electrocardiograms: A Numerical Study," Annals of Biomedical Engineering, 2009.

[2] H. Chen, K. K. Tseng, F. Zeng, H. N. Huang, and S. Y. Tu, "A New ECG Identification with Neural Network," IEEE Proc. of $201212^{\text {th }}$ International Conference on Hybrid Intelligent System (HIS), pp. 427430, December 2012.

[3] S. K. Mukhopadhyay, M. Mitra, and S. Mitra, "Time Plane ECG Feature Extraction using Hilbert Transform, Variable Threshold and Slope Reversal Approach," IEEE Proc. of 2011 International Conference on Communication and Industrial Application (ICCIA), pp. 1-4, December 2011.

[4] X. Qin, N. Zhang, and G. Hu, "A Novel Bézier Curve with Multiple Shape Parameters," IEEE Proc. of $20103^{\text {rd }}$ IEEE International Conference on Computer Science and Information Technology (ICCSIT), pp. 494-498, July 2010.

[5] P. Desyoo, S. Praesomboon, W. Sangpetch, and W. Suracherdkiati, "Discrete Mathematical Model for ECG Waveform using Kernel Function," IEEE Proc. of 2009 International Joint Conference ICROSSICE, pp. 5296-5300, August 2009.

[6] S. Mohapatra, "Classification of Electrocadiogram Waveforms using PNN," Department of Chemical Engineering, NIT Rourkela, Orissa, 2010.

[7] T. Ponghuttasilp, S. Sangthong, and S. Onriabroy, "On Design of Electrocardiograph Waveform by Mathematical Equations," Faculty of Engineering, King Mongkut's Institute of Technology Ladkrabang, 1998.

[8] W. Piboonwarangkun, P. Koosirivanichakom, and M. Sangworasil, "A Mathematical Model for Electrocardiograph Wave Form," Faculty of Engineering, King Mongkut's Institute of Technology Ladkrabang, 1989.

[9] V. Chutchavong, Linear-Phase Bernstein Filter for Equalized the Distorted Chrominance, LAP LAMBERT Academic Publishing: Germany, 2013.

[10] V. Chutchavong, O. Sangaroon, C. Benjangkaprasert, K. Janchipongvej, "Luminance-Chrominance Gain Equalizer Based on Bernstein Polynomials" IEEJ Trans. On Electronics, Information and Systems. Japan, vol. 130, No. 8, pp. 1411-1422, August 2010.

[11] A. Iglesias, "Computer-Aided Geometric Design and Computer Graphics: Bézier Curves and Surfaces," Department of Applied Mathematics and Computational Sciences, University of Cantabria, 2001.

[12] S. Wu, "Bernstein Polynomials, Bézier Curves, de Casteljiau's Algorithm," Computer Aided Geometric Design, Ferienakademie, 2004.

[13] B. T. Bertka, "An Introduction to Bézier Curves, B-Splines, and Tenser Product Surfaces with History and Applications," University of California Santa Cruz, 2008.

[14] P. J. Barendrecht, "A Gentle Introduction to Rational Bézier Curves and NURBS,” Eindhoven, June 7, 2012. 